\title{
Role of miRNAs in skeletal muscle aging
}

This article was published in the following Dove Press journal:

Clinical Interventions in Aging

\author{
Yan Zhengl,* \\ Jian Kongl,* \\ Qun $\mathrm{Li}^{2}$ \\ Yan Wang' \\ Jie $\mathrm{Li}^{\prime}$
}

'Department of Geriatrics, The First Hospital of Jilin University, Changchun I 3002 I, Jilin, China; ${ }^{2}$ Department of Thyroid Surgery, The First Hospital of Jilin University, Changchun I3002 I, Jilin, China

*These authors contributed equally to this work
Correspondence: Jie Li

Department of Geriatrics, The First Hospital of Jilin University, No 7I, Xinmin Street, Changchun I3002I, Jilin, China

Tel +8643I 88783989

Email jljieli_9@।63.com
Purpose: The aim of this study was to explore the role of miRNAs in the process of skeletal muscle aging.

Materials and methods: We analyzed the miRNA microarray datasets from 19 young and 17 old skeletal muscle samples by bioinformatic analysis. Differentially expressed miRNAs were identified, followed by function and pathway enrichment analysis. The expression of miRNAs were validated by real-time quantitative PCR (RT-qPCR) analysis.

Results: A total of 23 miRNAs were found to be differentially expressed in old muscle samples based on two platforms. Gene targets of upregulated miRNAs were significantly enriched in the oxytocin signaling pathway, AMP-activated protein kinase (AMPK) signaling pathway, and Notch signaling pathway. The target genes of downregulated miRNAs were significantly related to gap junction, salivary secretion, and estrogen signaling pathway. has-miR-19a and hsa-miR-34a were significant nodes in the miRNA regulatory network. has-miR-19a was closely related to the AMPK signaling pathway. hsa-miR-34a was closely related to cellular senescence and mitogen-activated protein kinase (MAPK) signaling pathway. PCR analysis showed that the expression of has-miR-34a-5p and has-miR-449b-5p was significantly higher in the patient group than in the control group, while no significant difference was observed in the expression of has-miR-19a-3p and has-miR-144-3p between the two groups. Furthermore, the expression of key target genes involved in cellular senescence (sirtuin 1 [SITRI]), MAPK signaling pathway (vascular endothelial growth factor A [VEGFA]), and AMPK signaling pathway (protein kinase AMP-activated catalytic subunit alpha 1 [PRKAA1] and 6-phosphofructo-2-kinase/fructose-2,6-biphosphatase 3 [PFKFB3]) were significantly increased in patients with sarcopenia.

Conclusion: has-miR-19a and hsa-miR-34a may play regulatory roles in the aging process of skeletal muscles and may be candidate targets to prevent muscle aging. Further experimental validations are warranted.

Keywords: skeletal muscle aging, differentially expressed miRNA, pathway, miRNA regulatory network

\section{Introduction}

Skeletal muscle aging (sarcopenia) is the key feature of age-related degenerative disease, which is characterized by skeletal muscle loss and function degeneration. Patients with sarcopenia present with $0.5 \%-1 \%$ muscle loss per year, which may be related to cachexia and other underlying malignant diseases. ${ }^{1}$ A previous study conducted in the UK among people with an average age of 67 years suggested that the incidence of sarcopenia was $4.6 \%$ in men and $7.9 \%$ in women. ${ }^{2}$ Another study reported that the incidence of sarcopenia was $36.5 \%$ in people with an average age of 70.1 years in the USA. ${ }^{3}$ As the population ages, the incidence of sarcopenia will be highlighted. Therefore, it is necessary to explore the mechanism of age-related sarcopenia.

Contributions have been made to characterize the mechanism associated with age-induced muscle loss. It is reported that age-related muscle loss and function reduction may be due to the abnormal production of aging cells and sensitivity to $\mathrm{BY} \mathrm{NC}$ and incorporate the Creative Commons Attribution - Non Commercial (unported, v3.0) License (http://creativecommons.org/licenses/by-nc/3.0/). By accessing the work you
hereby accept the Terms. Non-commercial uses of the work are permitted without any further permission from Dove Medical Press Limited, provided the work is properly attributed. For permission for commercial use of this work, please see paragraphs 4.2 and 5 of our Terms (https://www.dovepress.com/terms.php). 
cytokines. ${ }^{4}$ Mitochondrial dysfunction is an event in muscle aging and contributes to muscle mass loss and aerobic function decline. ${ }^{5}$ The superoxide generated by mitochondria is accumulated in aged cells and plays a contributory role in muscle aging. ${ }^{6}$ Muscle aging is associated with reduced $\mathrm{Ca}^{2+}$ homeostasis, and $\mathrm{Ca}^{2+}$ spark signaling appeared to be suppressed in aged skeletal muscles. ${ }^{7}$ In addition, Hall et $\mathrm{al}^{8}$ found that myofiber-associated satellite cells showed selfrenewal capabilities and the transplantation of these cells was proposed to prevent skeletal muscle aging. However, the mechanism of skeletal muscle aging has not yet been clarified. miRNAs are a class of small noncoding RNAs and have been reported to play a role in the aging process. ${ }^{9,10}$ Accumulating evidences have confirmed that miRNAs play a crucial role in the age-related changes in skeletal muscle mass, composition, and function. ${ }^{11-13}$ Moreover, some miRNAs are identified to be regulated with age and may be linked to key regulators of muscle protein synthesis and regeneration in young and old skeletal muscle. ${ }^{14}$ Despite these, the potential miRNAs involved in skeletal muscle aging have been fully reported.

In the present study, we analyzed the differentially expressed miRNAs between the young and old muscle samples, followed by function and pathway enrichment analysis. We aimed to explore the involvement of miRNAs in the potential mechanisms of muscle aging and provide new perspectives for the prevention of muscle aging.

\section{Materials and methods miRNA expression data}

The miRNA expression profile of human skeletal muscle (GSE23527) ${ }^{15}$ was downloaded from Gene Expression Omnibus (GEO) database. There were a total of 36 skeletal muscle samples: 19 from young male subjects and 17 from old male subjects. The data from 12 old and 12 young skeletal muscle samples were produced based on the GPL10358 platform, and the remaining data from 5 old and 7 young skeletal muscle samples were produced based on the GPL10415 platform.

\section{Data preprocessing and differentially expressed miRNA analysis}

The raw data were processed by limma package (version 3.10.3) ${ }^{16}$ in $\mathrm{R}$ (version 3.3.2), including background correction and expression profile normalization.

After preprocessing, differentially expressed miRNAs between the young and old samples based on the two platforms were analyzed by unpaired $t$-test through limma package, respectively. $P<0.05$ and $\log$ fold change $(\log$ FC) $>1$ were set as the cutoff values. All the differentially expressed miRNAs were pooled for further analysis.

\section{Prediction of miRNA targets}

The target genes of differentially expressed miRNAs were retrieved from DIANA-microT, MicroInspector, miRanda, MirTarget2, miTarget, NBmiRTar, PicTar, PITA, RNA22, RNAhybrid, and TargetScan/TargertScanS databases using the miRecords ${ }^{17}$ tool. The miRNA-target gene interactions deposited in at least five databases were collected. The miRNA-target gene network was constructed by Cytoscape version 3.2.0 ${ }^{18}$ (http://www.cytoscape.org/).

\section{Function and pathway enrichment analysis of miRNA targets}

Target genes of upregulated and downregulated miRNAs were subjected to Gene Ontology (GO) ${ }^{19}$ and Kyoto Encyclopedia of Genes and Genomes (KEGG) pathway ${ }^{20}$ enrichment analysis. The target genes were annotated to GO biological process (BP) terms by DAVID version 6.8 online tool ${ }^{21,22}$ (https://david.ncifcrf.gov/). The pathways that were closely related to miRNA targets were analyzed by clusterProfiler ${ }^{23}$ package. $P<0.05$ was set as the threshold value.

\section{Protein-protein interaction (PPI) network analysis}

The miRNA-target gene interactions were predicted by using the STRING database version $10.0^{24}$ (http://www.string-db. org/). The gene interaction pairs with the PPI score of $\geq 9$ were collected for PPI network construction using Cytoscape version 3.2.0. The significant modules with the score of $>10$ were mined using the MCODE plugin ${ }^{25}$ of the Cytoscape software. The module genes were subjected to KEGG pathway analysis with the criteria for significance set as $P<0.05$.

\section{Transcriptional factor (TF) prediction for target genes}

The TF-target gene interactions in the PPI network were predicted by iRegulon plugin version $1.3^{26}$ (http://apps.cytoscape. org/apps/iRegulon). Minimum identity between orthologous genes was set as 0.05 , and the maximum false discovery rate on motif similarity was set as 0.001 . The TF-gene interaction pairs with normalized enrichment score (NES) of $>4$ were used for TF regulatory network construction.

\section{Sample collection}

Five patients with sarcopenia and five cases without sarcopenia were enrolled from the First Hospital of the Jilin University. The skeletal muscle samples were collected via orthopedic 
surgery. All the patients provided written informed consent to participate in this study, and approval was obtained from the ethics committee of the First Hospital of Jilin University.

\section{RNA isolation and real-time quantitative PCR (RT-qPCR) analysis}

Total RNA was isolated from $100 \mathrm{mg}$ tissue samples using TRIZOL regent (Thermo Fisher Scientific, Waltham, MA, USA) for further analysis. RNA quality was determined by an Infinite M100 PRO microplate reader (Tecan, Crailsheim, Germany). cDNA was reverse transcribed from a total of $5 \mu \mathrm{g}$ of RNA by PrimeScript ${ }^{\mathrm{TM}}$ RT Master Mix, according to the manufacturer's instructions. RT-qPCR was performed, with a reaction volume of $20 \mu \mathrm{L}$, including $10 \mu \mathrm{L}$ of the SYBR Premix EX Taq $(2 \times), 1 \mu \mathrm{L}$ of forward primer, $1 \mu \mathrm{L}$ of reverse primer, and $8 \mu \mathrm{L}$ of cDNA. The primer sequences are summarized in Table 1. The PCR reaction conditions used were as follows: $50.0^{\circ} \mathrm{C}$ for 3 minutes, $95.0^{\circ} \mathrm{C}$ for 3 minutes, followed by 40 cycles of $95.0^{\circ} \mathrm{C}$ for 10 seconds and $60.0^{\circ} \mathrm{C}$ for 30 seconds.

\section{Statistical analyses}

All the data were expressed as mean \pm SD and analyzed by the SPSS 22.0 software (IBM Corporation, Armonk, NY, USA). $P<0.05$ was considered significant.

\section{Results \\ Differentially expressed miRNAs}

Based on the GPL10358 platform, there were 12 differentially expressed miRNAs between the young and old samples, including six downregulated miRNAs and six upregulated miRNAs. In addition, 11 miRNAs were found to be differentially expressed between the young and old samples based on the GPL10415 platform, including five upregulated miRNAs and six downregulated miRNAs (Table 2). All the differentially expressed miRNAs were pooled for further analysis.

\section{miRNA-target gene regulatory network}

A total of 1,236 miRNA-target gene interaction pairs were obtained through miRecords, which contained six upregulated miRNAs (hsa-miR-34a, hsa-miR-449b, hsa-miR-215, hsa-miR-499-5p, hsa-miR-184, and hsa-miR-450a) and six downregulated miRNAs (hsa-miR-19a, hsa-miR-144, hsa-miR532-5p, hsa-miR-365, hsa-miR-487b, and hsa-miR-362-5p). The miRNA-target gene regulatory network is illustrated in Figure 1. The significant nodes in this network included hsa-miR-19a (degree $=434$ ), hsa-miR-34a (degree $=217$ ), hsamiR-144 (degree $=227)$, and hsa-miR-532-5p (degree $=166$ ). Significant pathways that were closely related to significant miRNAs are shown in Figure 2. Notably, has-miR-19a was significantly enriched in the AMP-activated protein kinase (AMPK) signaling pathway (protein kinase AMP-activated catalytic subunit alpha 1 [PRKAA1] and 6-phosphofructo2-kinase/fructose-2,6-biphosphatase 3 [PFKFB3]), and hasmiR-34a was significantly enriched in cellular senescence (sirtuin 1 [SITRI] and cell division cycle 25A [CDC25A]) and mitogen-activated protein kinase (MAPK) signaling pathway (vascular endothelial growth factor A [VEGFA] and microtubule associated protein tau [MAPT]).

\section{Significant function and pathways related to miRNA targets}

The target genes of upregulated miRNAs were significantly enriched in 30 pathways, such as the oxytocin signaling pathway, AMPK signaling pathway, and Fc gamma R-mediated phagocytosis. The downregulated miRNAs were significantly enriched in 67 pathways, such as gap junction, estrogen signaling pathway, and prolactin signaling pathway. The top 20 significant pathways for miRNA-target genes are shown in Figure 3.

With the criterion for significance set as $P<0.05,70$ GO-BP terms were found to be closely related to gene targets of upregulated miRNAs, while 143 GO functions were

Table I Primer sequences for PCR analysis

\begin{tabular}{l|l}
\hline Symbol & Sequences $\left(\mathbf{5}^{\prime} \mathbf{-} \mathbf{3}^{\prime} \mathbf{)}\right.$ \\
\hline Human-U6-RT & GTCGTATCCAGTGCAGGGTCCGAGGTATTCGCACTGGATACGACAAAATATG \\
General reverse primer & GTGCAGGGTCCGAGGT \\
hsa-miR-19a-3p-RT & GTCGTATCCAGTGCAGGGTCCGAGGTATTCGCACTGGATACGACTCAGTT \\
hsa-miR-I44-3p-RT & GTCGTATCCAGTGCAGGGTCCGAGGTATTCGCACTGGATACGACAGTACA \\
hsa-miR-34a-5p-RT & GTCGTATCCAGTGCAGGGTCCGAGGTATTCGCACTGGATACGACACAACC \\
has-miR-532-5p-RT & GTCGTATCCAGTGCAGGGTCCGAGGTATTCGCACTGGATACGACACGGTC \\
hsa-miR-449b-5p-RT & GTCGTATCCAGTGCAGGGTCCGAGGTATTCGCACTGGATACGACGCCAGC \\
JH-hsa-miR-19a-3p-F & GCGCTGTGCAAATCTATGCAA \\
JH-hsa-miR-I44-3p-F & GCGCGCTACAGTATAGATGA \\
JH-hsa-miR-34a-5p-F & GCTGGCAGTGTCTTAGCT \\
JH-has-miR-532-5p-F & GCCATGCCTTGAGTGTAG \\
JH-hsa-miR-449b-5p-F & GCGCAGGCAGTGTATTGTTA \\
\hline
\end{tabular}


Table 2 Differentially expressed miRNAs

\begin{tabular}{|c|c|c|c|c|c|}
\hline \multicolumn{3}{|c|}{ GSE23527-GPLI 0358} & \multicolumn{3}{|c|}{ GSE23527-GPLI $04 \mid 5$} \\
\hline miRNA & $\log$ FC & $P$-value & miRNA & Log FC & $P$-value \\
\hline hsa-miR-450a & 1.513142 & $9.77 \mathrm{E}-05$ & hsa-miR-34a & $1.93873 \mid 4$ & 0.000656 \\
\hline hsa-miR-I6-2* & I.183442 & $1.66 \mathrm{E}-03$ & hsa-miR-548j & 1.4707223 & 0.001875 \\
\hline hsa-miR-2I5 & 1.153009 & 4.86E-04 & hsa-miR-933 & I.I785806 & 0.001847 \\
\hline hsa-miR-I84 & 1.093055 & $9.16 \mathrm{E}-04$ & hsa-miR-449b & 1.164987 & 0.003803 \\
\hline hsa-miR-374b* & 1.090489 & $8.31 \mathrm{E}-04$ & hsa-miR-499-5p & I.1096546 & 0.00423 \\
\hline hsa-miR-I5b* & 1.017695 & $3.8 \mathrm{IE}-03$ & hsa-miR-|27| & -1.0073725 & 0.006553 \\
\hline hsa-miR-500* & -1.021664 & $1.65 \mathrm{E}-03$ & hsa-miR-I303 & -1.2463053 & 0.009014 \\
\hline hsa-miR-24-2* & -1.034437 & $3.63 \mathrm{E}-04$ & hsa-miR-7-2* & -1.2652065 & 0.000861 \\
\hline hsa-miR-362-5p & -1.083984 & $4.29 \mathrm{E}-03$ & hsa-miR-365 & -1.3649855 & 0.008124 \\
\hline hsa-miR-487b & -1.097873 & $4.66 \mathrm{E}-05$ & hsa-miR-19a & -1.6839475 & 0.006358 \\
\hline hsa-miR-532-5p & -1.161705 & I.66E-04 & hsa-miR-I44 & -2.5983958 & 0.000196 \\
\hline hsa-miR-362-3p & -1.196579 & $8.39 \mathrm{E}-04$ & & & \\
\hline
\end{tabular}

Note: *Indicates the miRNA with lower expression level.

Abbreviations: FC, fold change. In general, the two arms of a miRNA precursor are likely to produce two mirnas separately.

related to target genes of downregulated miRNAs. As shown in Figure 4, the top 20 significant BP terms for upregulated miRNAs included the regulation of synaptic vesicle exocytosis, Notch signaling pathway, and histone deacetylation.
The top 20 significant BP terms for downregulated miRNAs comprised the regulation of ventricular cardiac muscle cell membrane, response to muscle stretch, and cardiac muscle cell action potential involved in contraction.

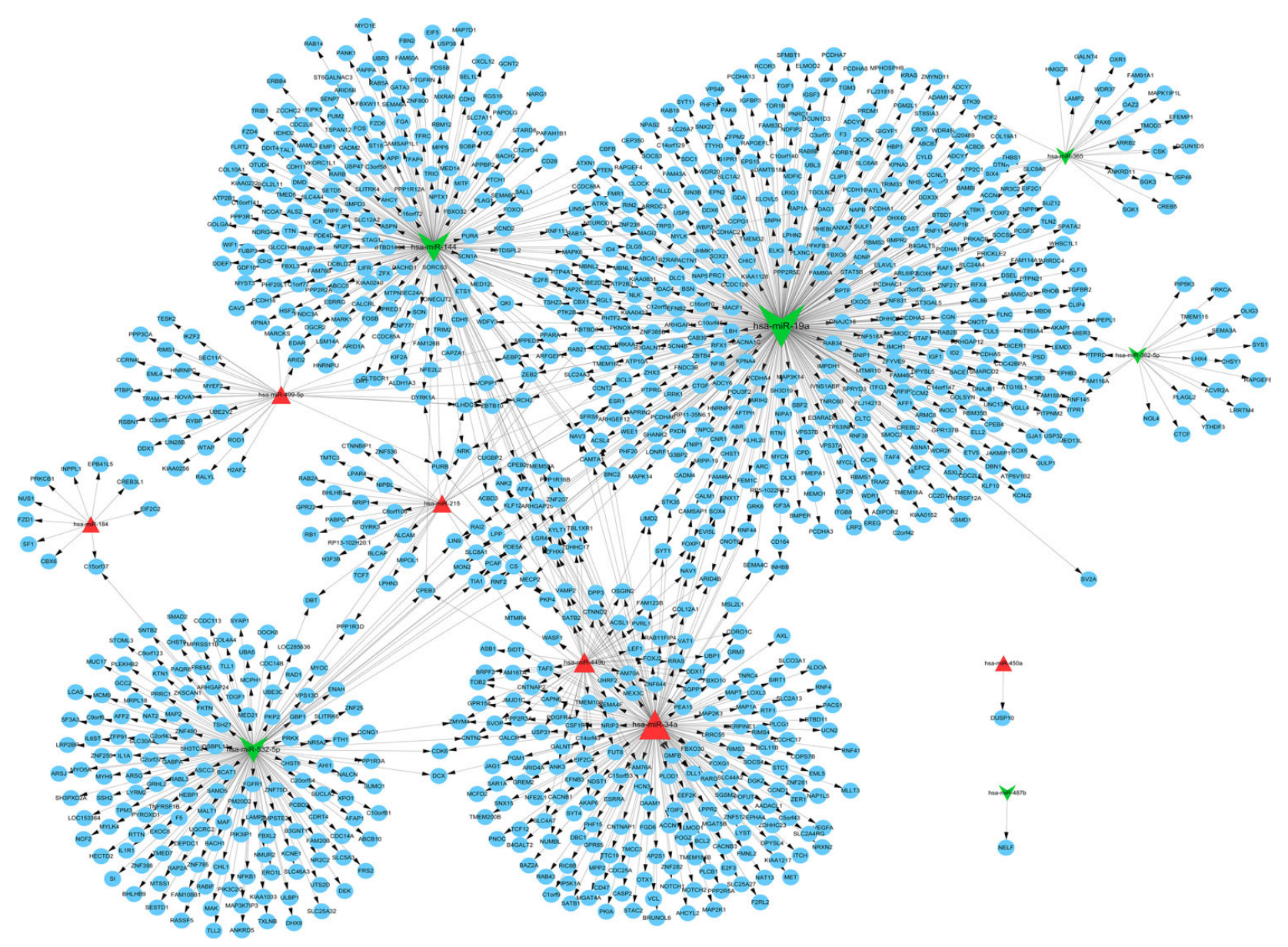

Figure I miRNA-target gene regulatory network.

Notes: Red triangle represents the upregulated miRNAs, green arrow represents the downregulated miRNAs, and blue circle represents the target genes. The black line with arrows indicates the interaction between miRNAs and target genes. 


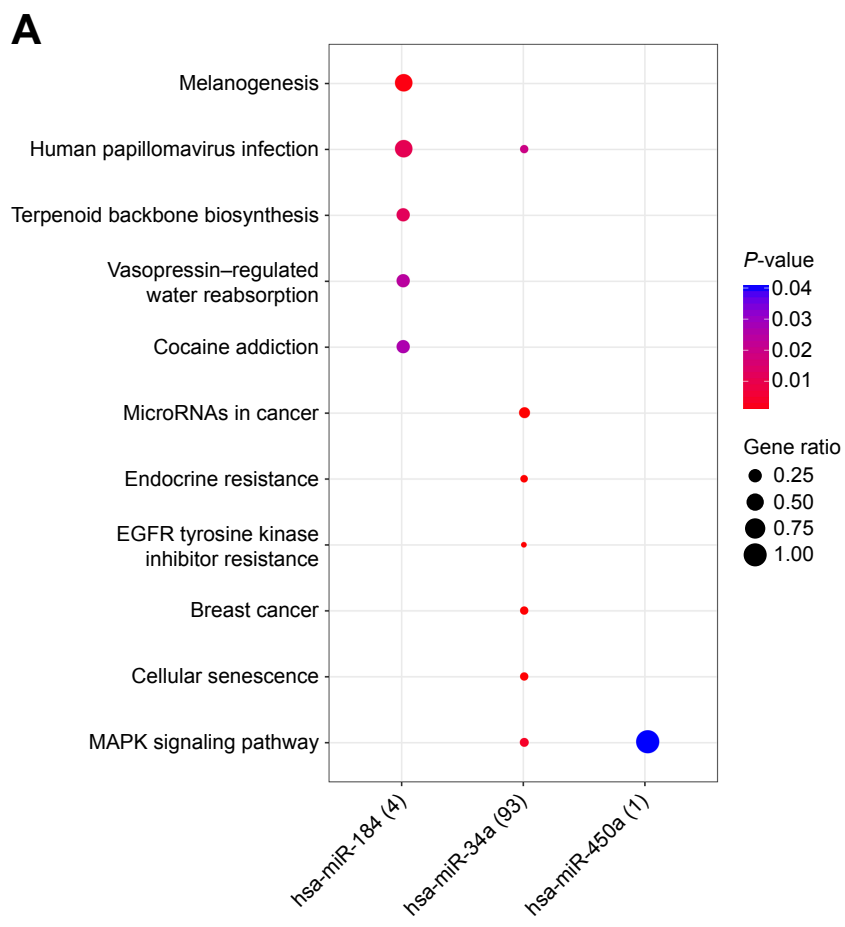

Figure 2 The significant pathways related to miRNAs.

Notes: (A) For upregulated miRNAs. (B) For downregulated miRNAs.

Abbreviations: DCM, dilated cardiomyopathy; IBD, inflammatory bowel disease.

\section{PPI network and module analysis}

A PPI network of target genes was constructed with 1,291 edges connecting with 414 nodes. On the basis of score of $>10$, three significant modules were screened, including module-a (score $=22$, containing 15 nodes and 105 edges), module-b (score $=15$, containing 15 nodes and 105 edges), and module-c (score $=12$, containing 12 nodes and 66 interaction pairs; Figure 5).

Genes in module-a were significantly enriched in two pathways, including ubiquitin-mediated proteolysis and circadian rhythm. Similarly, two pathways were closely related to module-b genes, including endocytosis and synaptic vesicle cycle. A total of 36 pathways were significantly enriched by module-c genes, such as retrograde endocannabinoid signaling, glutamatergic synapse, ovarian steroidogenesis, and regulation of lipolysis in adipocytes (Table 3 ).

\section{TF regulatory network}

A total of $344 \mathrm{TF}$-target gene regulatory interactions were predicted, including 58 upregulated miRNA-target genes, 149 downregulated miRNA-target genes, and 2 TFs (early growth response 1 [EGR1] and nanos C2HC-type zinc finger 1 [NANOS1]; Figure 6).

\section{Subjects}

Subjects (two females and three males) with a mean age of $37.6 \pm 14.5$ years were included in the control group for

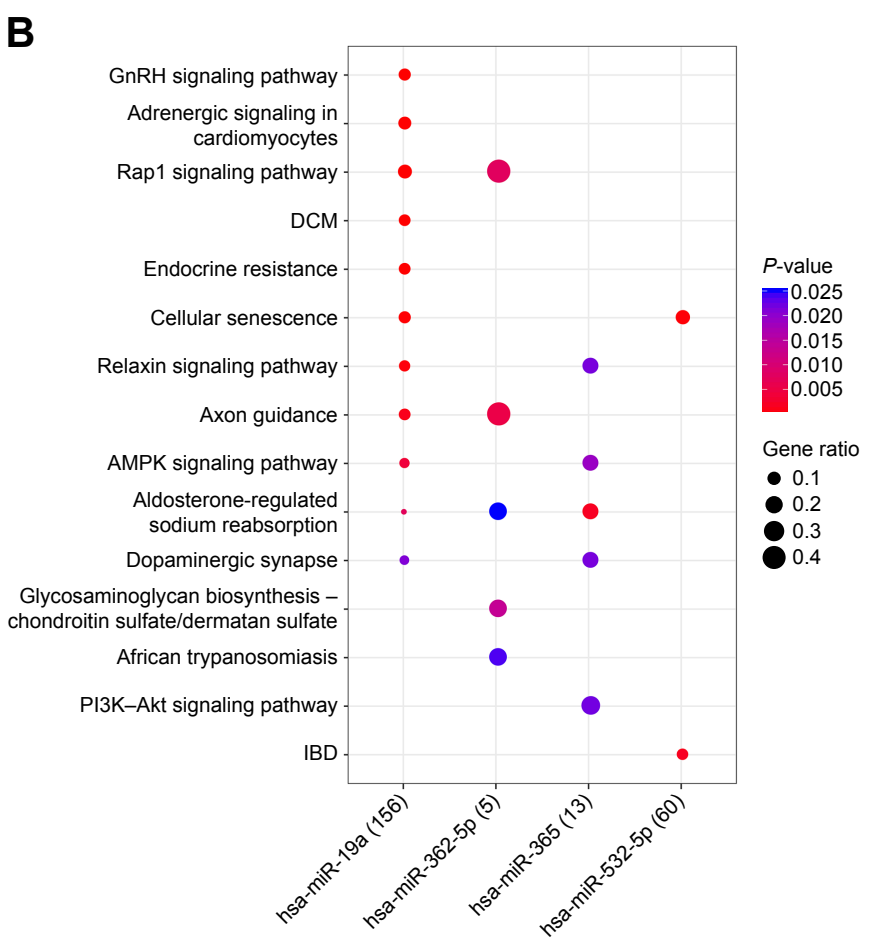

fractures. The gender-paired patients with a mean age of $82.4 \pm 3.3$ years were included in the patient group.

\section{RT-qPCR validation}

The expression of has-miR-19a-3p, has-miR-144-3p, hasmiR-34a-5p, and has-miR-449b-5p were measured by PCR analysis. Results showed that the expression of hasmiR-34a-5p and has-miR-449b-5p was significantly higher in the patient group than in the control group, which was consistent with the analysis described earlier $(P<0.01)$. There was no significant difference in the expression of has-miR-19a-3p and has-miR-144-3p between the two groups, which was not consistent with the bioinformatic prediction (Figure 7).

Furthermore, the expression of key target genes involved in cellular senescence (SIRT1 and CDC25A), MAPK signaling pathway (VEGFA and MAPT), and AMPK signaling pathway (PRKAA1 and PFKFB3) was also detected. Results showed that the expression levels of SIRT1, VEGFA, PRKAA1, and PFKFB3 were significantly increased in the patient group compared to that in the control group $(P<0.05)$; however, CDC25A and MAPT expression did not exhibit significant difference between the two groups (Figure 8).

\section{Discussion}

The role of miRNAs in regulating the aging process has not yet been elucidated. In a previous study, the microarray 
A

hsa04921:oxytocin signaling pathway
hsa04152:AMPK signaling pathway

hsa04666:Fc gamma R-mediated phagocytosis hsa05216:thyroid cancer

hsa04919:thyroid hormone signaling pathway hsa04015:Rap1 signaling pathway hsa04330:Notch signaling pathway hsa05231:choline metabolism in cancer hsa05205:proteoglycans in cancer hsa04070:phosphatidylinositol signaling system hsa05219:bladder cancer hsa05212:pancreatic cancer hsa05200:pathways in cancer hsa04310:Wnt signaling pathway hsa05218:melanoma hsa04320:dorsoventral axis formation hsa05214:glioma hsa05223:non-small-cell lung cancer hsa05206:microRNAs in cancer hsa05215:prostate cancer

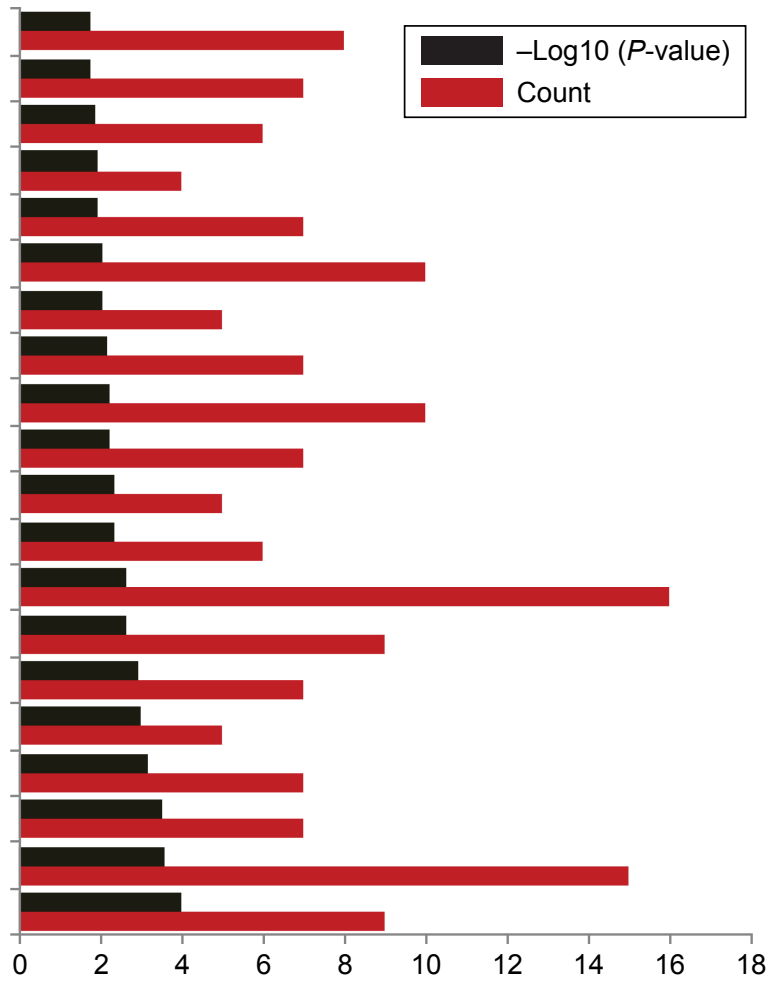
hsa04540:gap junction
hsa04970:salivary secretion
hsa04915:estrogen signaling pathway
hsa04917:prolactin signaling pathway
hsa04114:oocyte meiosis
hsa04015:Rap1 signaling pathway
hsa04270:vascular smooth muscle contraction
hsa04720:long-term potentiation
hsa04912:GnRH signaling pathway
hsa04611:platelet activation
hsa04914:progesterone-mediated oocyte maturation
hsa04971: gastric acid secretion
hsa05414:dilated cardiomyopathy
hsa04022:cGMP-PKG signaling pathway
hsa04068:FoxO signaling pathway
hsa04921:oxytocin signaling pathway
hsa04024:cAMP signaling pathway
hsa04550:signaling pathways regulating...
hsa05205:proteoglycans in cancer hsa04261:adrenergic signaling in cardiomyocytes

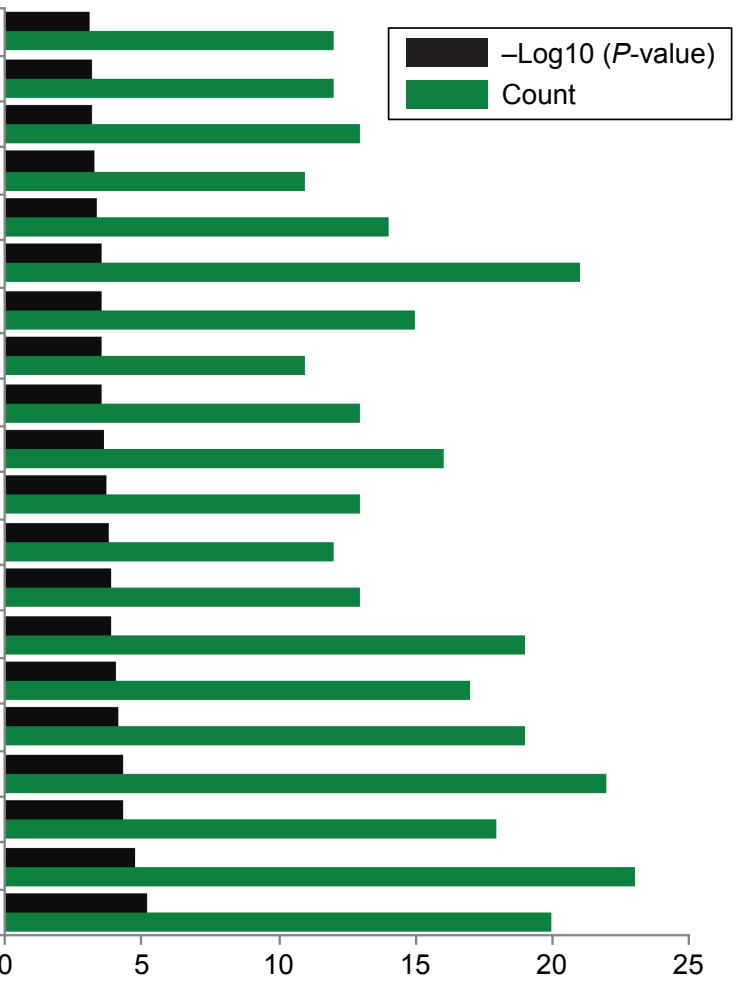

Figure 3 Top 20 significant pathways enriched by gene targets.

Notes: (A) Results of upregulated miRNAs. (B) Results of downregulated miRNAs.

data (GSE23527) were generated to analyze differentially expressed miRNAs in aging human skeletal muscle, followed by the identification of key cellular functions and canonical pathways related to the progression of muscle loss with aging (sarcopenia) based on ingenuity pathway analysis; the results showed that aging is characterized by an enhanced expression of Let-7 family members that may downregulate genes related to cellular proliferation. ${ }^{15}$ Compared to this study, 
A

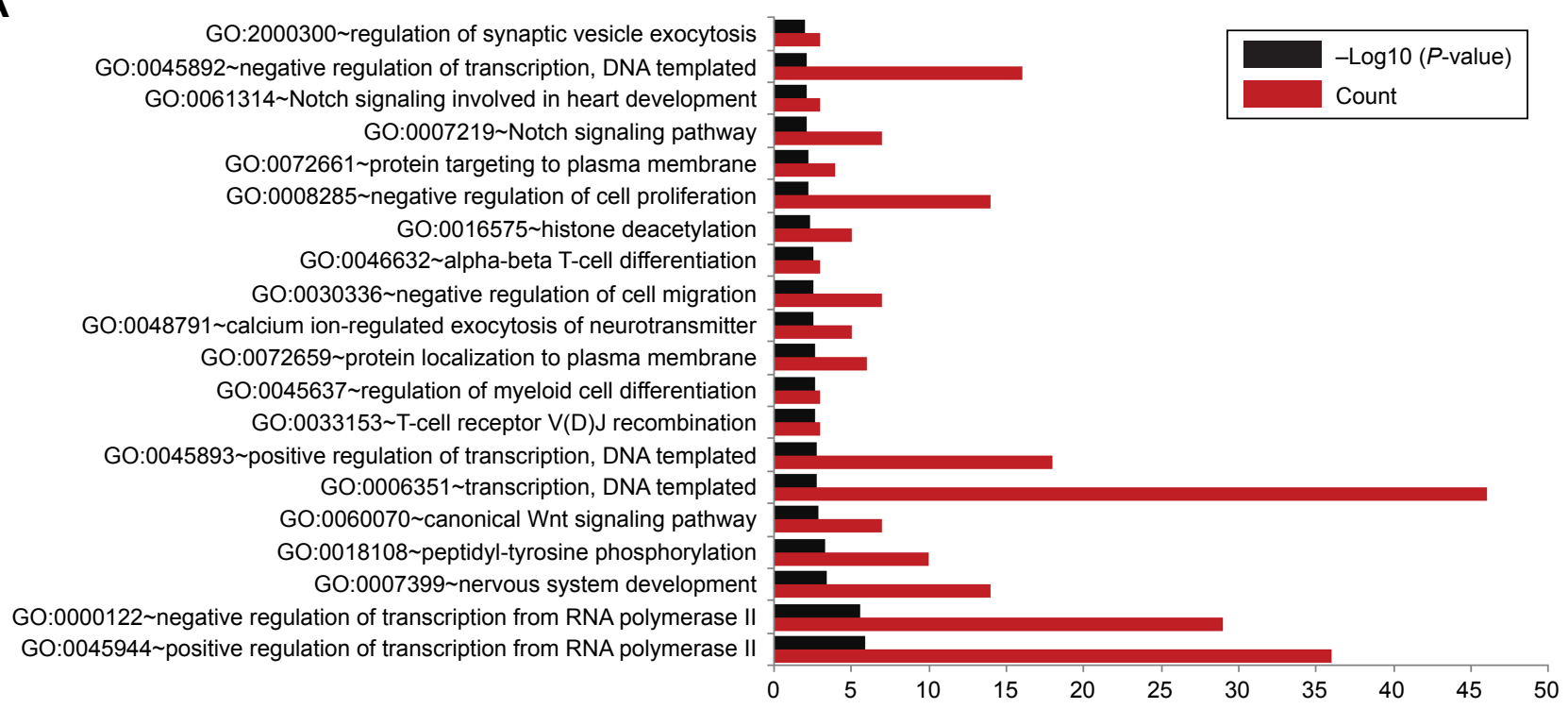

B

GO:0060307 regulation of ventricular cardiac muscle cell membrane. GO:0045892 negative regulation of transcription, DNA templated GO:0035994 response to muscle stretch GO:0086002 cardiac muscle cell action potential involved in contraction GO:0045727 positive regulation of translation GO:0001701 in utero embryonic development GO:0010628 positive regulation of gene expression GO:0010629 negative regulation of gene expression GO:0007420 brain development GO:0001764 neuron migration GO:0007156 homophilic cell adhesion via plasma membrane adhesion GO:0007155 cell adhesion GO:0008285 negative regulation of cell proliferation GO:0006351 transcription, DNA templated GO:0007264 small GTPase mediated signal transduction GO:0007399 nervous system development GO:0000122 negative regulation of transcription from RNA polymerase II GO:0006366 transcription from RNA polymerase II promoter GO:0045893 positive regulation of transcription, DNA templated GO:0045944 positive regulation of transcription from RNA polymerase II

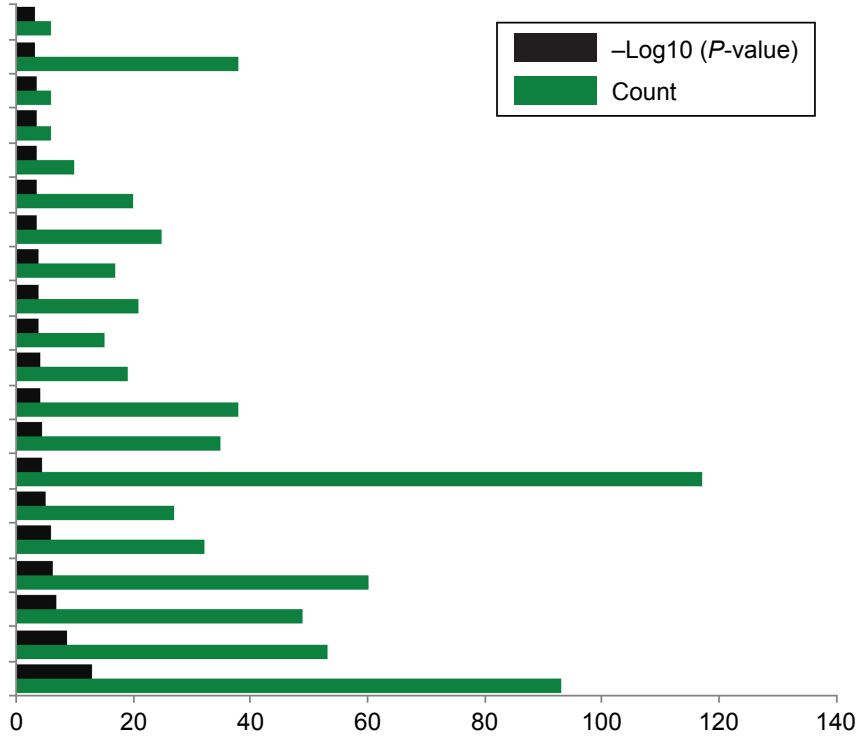

Figure 4 Top 20 biological processes enriched by target genes.

Notes: (A) Results of upregulated miRNAs. (B) Results of downregulated miRNAs. Abbreviation: GO, Gene Ontology.

we downloaded this microarray data and also identified the differentially expressed miRNAs between young and old skeletal muscles. Furthermore, function and pathway enrichment analyses were also performed. In addition to these, we conducted other analyses, such as PPI network and TF prediction for target genes. These comprehensive analyses will help us identify more significant miRNAs. The results suggested that a total of 23 miRNAs were differentially expressed in old muscle samples, among which 12 were involved in the miRNA-target gene network.

Pathway analysis showed that the gene targets of upregulated miRNAs were significantly enriched in the AMPK signaling pathway. It is reported that AMPK plays a key role in controlling the aging process by regulating energy metabolism, autophagic degradation, and stress resistance. ${ }^{27}$ AMPK can improve cellular stress resistance and play a critical role in suppressing cell aging. The loss of AMPK activation increased cell stress and suppressed autophagic clearance, which contributed to the cell aging process. ${ }^{27}$ Another study found that the activation of AMPK signaling was reduced in the skeletal muscles of old rats, suggesting that AMPK activation reduction was a contributory factor in aging process. ${ }^{28}$ Furthermore, it is reported that deletion of muscle-specific PRKAA1 may lead to delayed development 

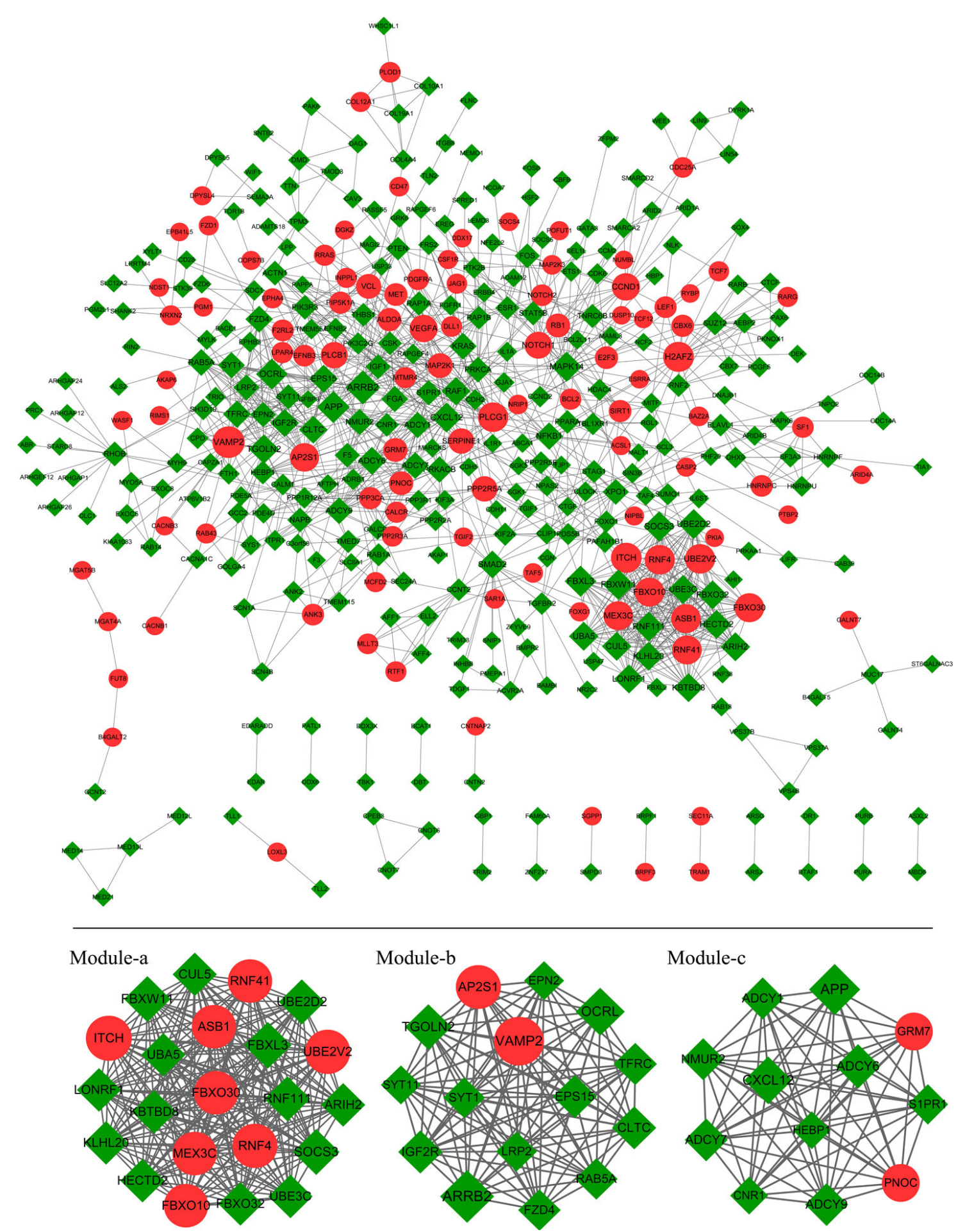

Figure 5 PPI network of target genes.

Notes: Green rhombus nodes represent the target genes of downregulated miRNAs. Red circular nodes represent the target genes of upregulated miRNAs. The edges indicate the interaction between target genes.

Abbreviation: PPI, protein-protein interaction.

of skeletal muscles and regulate lipid metabolism and mitochondrial oxidation in mice fed a high-fat diet, suggesting that PRKAA1 may be involved in related metabolic diseases. ${ }^{29}$ In this study, has-miR-19a, with a degree of 434, was the most significant node in the miRNA-target gene network. Pathway analysis also showed that has-miR-19a was closely related to the AMPK signaling pathway. The expression of PRKAA1 and PFKFB3 involved in the AMPK signaling pathway was significantly increased in the patients with sarcopenia. All these indicated that AMPK signaling played a critical role in 
Table 3 Significant pathways that enriched by module genes

\begin{tabular}{|c|c|c|c|c|}
\hline & KEGG pathway & Count & $P$-value & Genes \\
\hline \multirow[t]{2}{*}{ Module-a } & hsa04 I 20:ubiquitin-mediated proteolysis & 6 & I.52E-07 & CUL5, UBE2D2, SOCS3, UBE3C, ITCH, FBXWII \\
\hline & hsa047 I0:circadian rhythm & 2 & 3.53E-02 & FBXWII, FBXL3 \\
\hline \multirow[t]{2}{*}{ Module-b } & hsa04|44:endocytosis & 8 & $6.30 \mathrm{E}-08$ & $\begin{array}{l}\text { EPSI5, TFRC, ARRB2, IGF2R, AP2SI, RAB5A, } \\
\text { CLTC, EPN2 }\end{array}$ \\
\hline & hsa0472I:synaptic vesicle cycle & 4 & I.50E-04 & SYTI, AP2SI, VAMP2, CLTC \\
\hline \multirow[t]{36}{*}{ Module-c } & hsa04723:retrograde endocannabinoid signaling & 5 & $5.12 \mathrm{E}-06$ & ADCYI, ADCY7, ADCY9, CNRI, ADCY6 \\
\hline & hsa04724:glutamatergic synapse & 5 & $8.31 \mathrm{E}-06$ & ADCYI, ADCY7, ADCY9, GRM7, ADCY6 \\
\hline & hsa049|3:ovarian steroidogenesis & 4 & $2.73 \mathrm{E}-05$ & ADCYI, ADCY7, ADCY9, ADCY6 \\
\hline & hsa04923:regulation of lipolysis in adipocytes & 4 & 4.09E-05 & ADCYI, ADCY7, ADCY9, ADCY6 \\
\hline & hsa04062:chemokine signaling pathway & 5 & $5.76 \mathrm{E}-05$ & ADCYI, ADCY7, ADCY9, ADCY6, CXCLI2 \\
\hline & hsa04976:bile secretion & 4 & 7.67E-05 & ADCYI, ADCY7, ADCY9, ADCY6 \\
\hline & hsa04918:thyroid hormone synthesis & 4 & $8.0 I E-05$ & ADCYI, ADCY7, ADCY9, ADCY6 \\
\hline & hsa0497l:gastric acid secretion & 4 & $9.08 \mathrm{E}-05$ & ADCYI, ADCY7, ADCY9, ADCY6 \\
\hline & hsa040 I5:Rap I signaling pathway & 5 & $9.27 \mathrm{E}-05$ & ADCYI, ADCY7, ADCY9, CNRI, ADCY6 \\
\hline & hsa04925:aldosterone synthesis and secretion & 4 & 1.24E-04 & ADCYI, ADCY7, ADCY9, ADCY6 \\
\hline & hsa054 I4:dilated cardiomyopathy & 4 & $1.38 \mathrm{E}-04$ & ADCYI, ADCY7, ADCY9, ADCY6 \\
\hline & hsa04727:GABAergic synapse & 4 & I.43E-04 & ADCYI, ADCY7, ADCY9, ADCY6 \\
\hline & hsa049II:insulin secretion & 4 & $1.43 \mathrm{E}-04$ & ADCYI, ADCY7, ADCY9, ADCY6 \\
\hline & hsa04970:salivary secretion & 4 & I. $48 \mathrm{E}-04$ & ADCYI, ADCY7, ADCY9, ADCY6 \\
\hline & hsa049 |4:progesterone-mediated oocyte maturation & 4 & I.53E-04 & ADCYI, ADCY7, ADCY9, ADCY6 \\
\hline & hsa04540:gap junction & 4 & I.59E-04 & ADCYI, ADCY7, ADCY9, ADCY6 \\
\hline & hsa049/2:GnRH signaling pathway & 4 & I.75E-04 & ADCYI, ADCY7, ADCY9, ADCY6 \\
\hline & hsa05032:morphine addiction & 4 & I.75E-04 & ADCYI, ADCY7, ADCY9, ADCY6 \\
\hline & hsa04972:pancreatic secretion & 4 & I.87E-04 & ADCYI, ADCY7, ADCY9, ADCY6 \\
\hline & hsa047/3:circadian entrainment & 4 & $1.99 \mathrm{E}-04$ & ADCYI, ADCY7, ADCY9, ADCY6 \\
\hline & hsa04750:inflammatory mediator regulation of TRP channels & 4 & 2.18E-04 & ADCYI, ADCY7, ADCY9, ADCY6 \\
\hline & hsa04915:estrogen signaling pathway & 4 & $2.25 \mathrm{E}-04$ & ADCYI, ADCY7, ADCY9, ADCY6 \\
\hline & hsa04916:melanogenesis & 4 & $2.32 \mathrm{E}-04$ & ADCYI, ADCY7, ADCY9, ADCY6 \\
\hline & hsa04II4:oocyte meiosis & 4 & 2.99E-04 & ADCYI, ADCY7, ADCY9, ADCY6 \\
\hline & hsa04725:cholinergic synapse & 4 & $3.16 \mathrm{E}-04$ & ADCYI, ADCY7, ADCY9, ADCY6 \\
\hline & hsa04270:vascular smooth muscle contraction & 4 & 3.88E-04 & ADCYI, ADCY7, ADCY9, ADCY6 \\
\hline & hsa046 I I:platelet activation & 4 & $5.03 \mathrm{E}-04$ & ADCYI, ADCY7, ADCY9, ADCY6 \\
\hline & hsa0426I:adrenergic signaling in cardiomyocytes & 4 & 7.07E-04 & ADCYI, ADCY7, ADCY9, ADCY6 \\
\hline & hsa0492I:oxytocin signaling pathway & 4 & $8.90 \mathrm{E}-04$ & ADCYI, ADCY7, ADCY9, ADCY6 \\
\hline & hsa04022:cGMP-PKG signaling pathway & 4 & $1.03 E-03$ & ADCYI, ADCY7, ADCY9, ADCY6 \\
\hline & hsa05200:pathways in cancer & 5 & $1.03 \mathrm{E}-03$ & ADCYI, ADCY7, ADCY9, ADCY6, CXCLI2 \\
\hline & hsa00230:purine metabolism & 4 & $1.22 \mathrm{E}-03$ & ADCYI, ADCY7, ADCY9, ADCY6 \\
\hline & hsa04024:cAMP signaling pathway & 4 & I.7IE-03 & ADCYI, ADCY7, ADCY9, ADCY6 \\
\hline & hsa05I66:HTLV-I infection & 4 & $3.58 \mathrm{E}-03$ & ADCYI, ADCY7, ADCY9, ADCY6 \\
\hline & hsa04080:neuroactive ligand-receptor interaction & 4 & 4.47E-03 & SIPRI, NMUR2, GRM7, CNRI \\
\hline & hsa04020:calcium signaling pathway & 3 & $2.13 E-02$ & ADCYI, ADCY7, ADCY9 \\
\hline
\end{tabular}

Abbreviations: KEGG, Kyoto Encyclopedia of Genes and Genomes; TRP, transient receptor potential.

muscle aging process involved with has-miR-19a. hsa-miR$34 a$, with a degree of 217 , was another significant node in the miRNA-target gene network. Pathway analysis indicated that has-miR-34a was significantly enriched in cellular senescence and MAPK signaling pathway. It is reported that cellular senescence may be involved in the aging process via the accumulation of senescent cells and stem cell loss. ${ }^{30} \mathrm{Cel}-$ lular senescence may be a marker for the aging process, suggesting that the pathway results were significant. In addition, MAPK plays a critical role in regulating stress responses in skeletal muscles. The activation of MAPK can be induced by exercise, which is involved in preventing skeletal muscle atrophy. ${ }^{31} \mathrm{p} 38$ MAPK signaling pathway affects the autonomous loss and renewal of stem cells. ${ }^{32}$ The satellite cell homeostatic process was dysregulated by p38 MAPK signaling in the skeletal muscles of aged mice. ${ }^{32}$ Moreover, we found that the expression levels of SIRT1 involved in cellular senescence and VEGFA involved in MAPK signaling pathway were significantly increased in patients with sarcopenia. Aging gastric mucosa has significantly reduced 


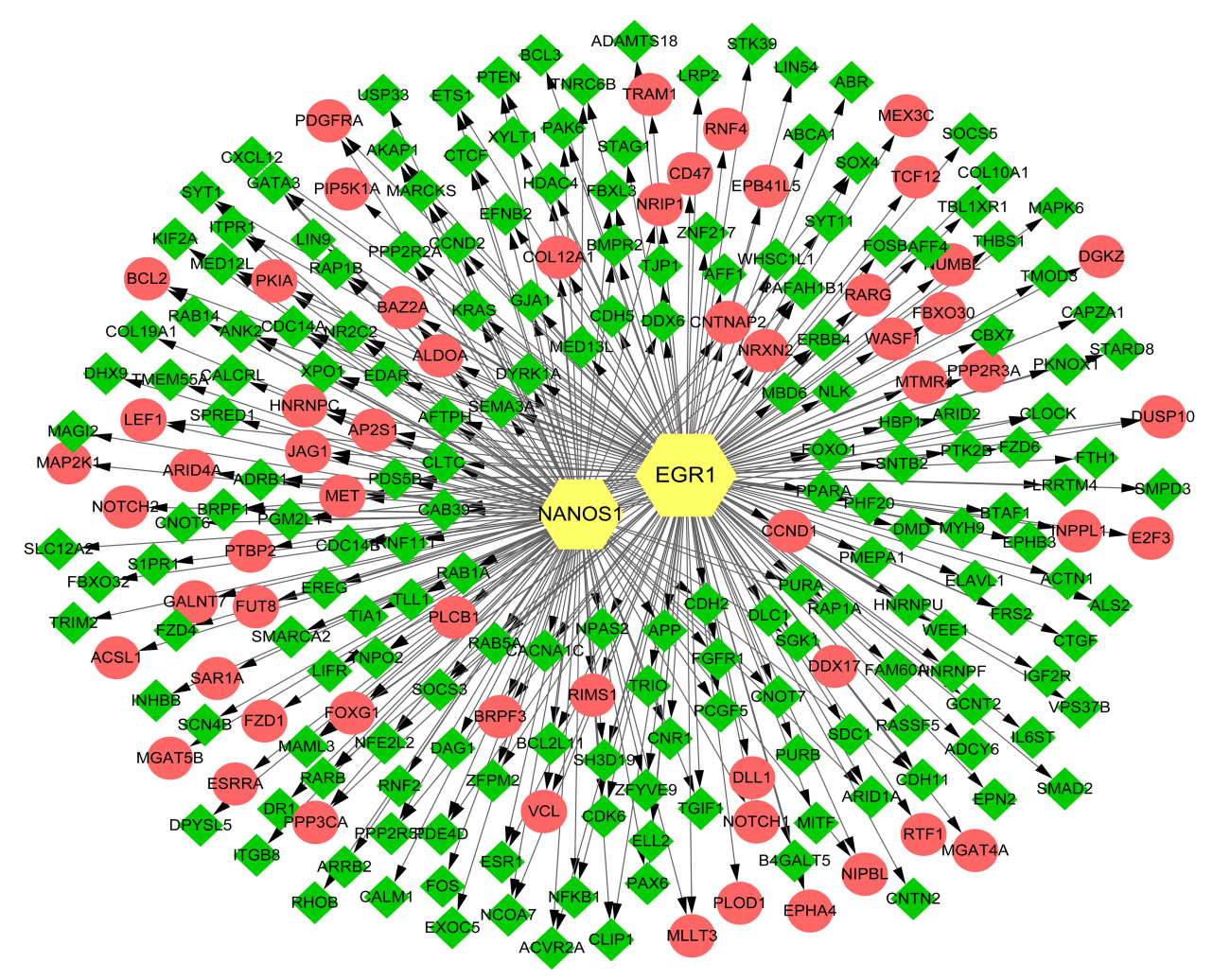

Figure 6 TF regulatory network.

Notes: Green, downregulated miRNAs. Red, upregulated miRNAs. Yellow, TFs. Abbreviation: TF, transcriptional factor.
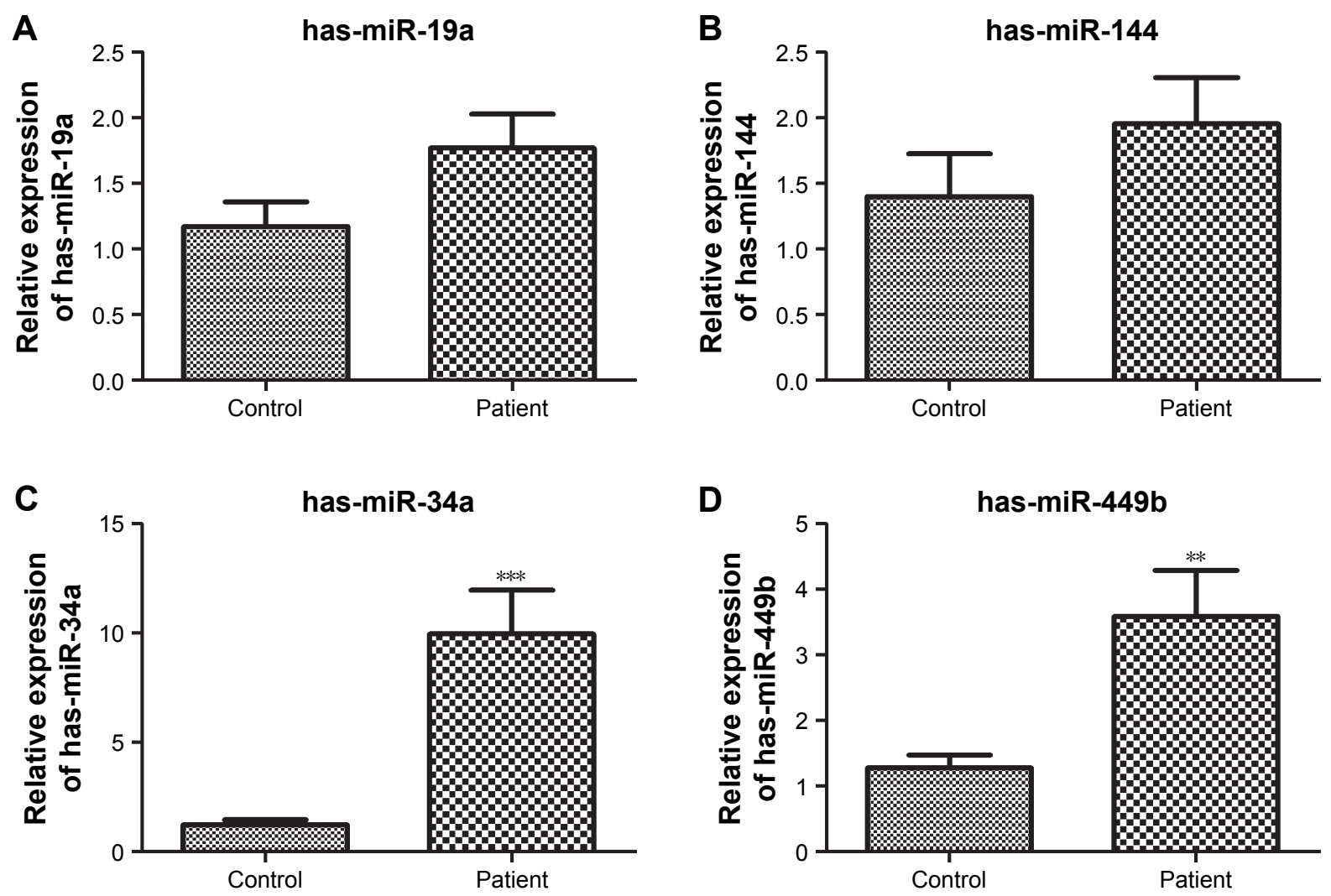

Figure 7 RT-qPCR analyzed the expression of has-miR-19a-3p, has-miR-144-3p, has-miR-34a-5p, and has-miR-449b-5p.

Notes: (A) The relative expression of has-miR-19a. (B) The relative expression of has-miR-144. (C) The relative expression of has-miR-34a. (D) The relative expression of has-miR-449b. $* * P<0.01, * * * P<0.001$.

Abbreviation: RT-qPCR, real-time quantitative PCR. 

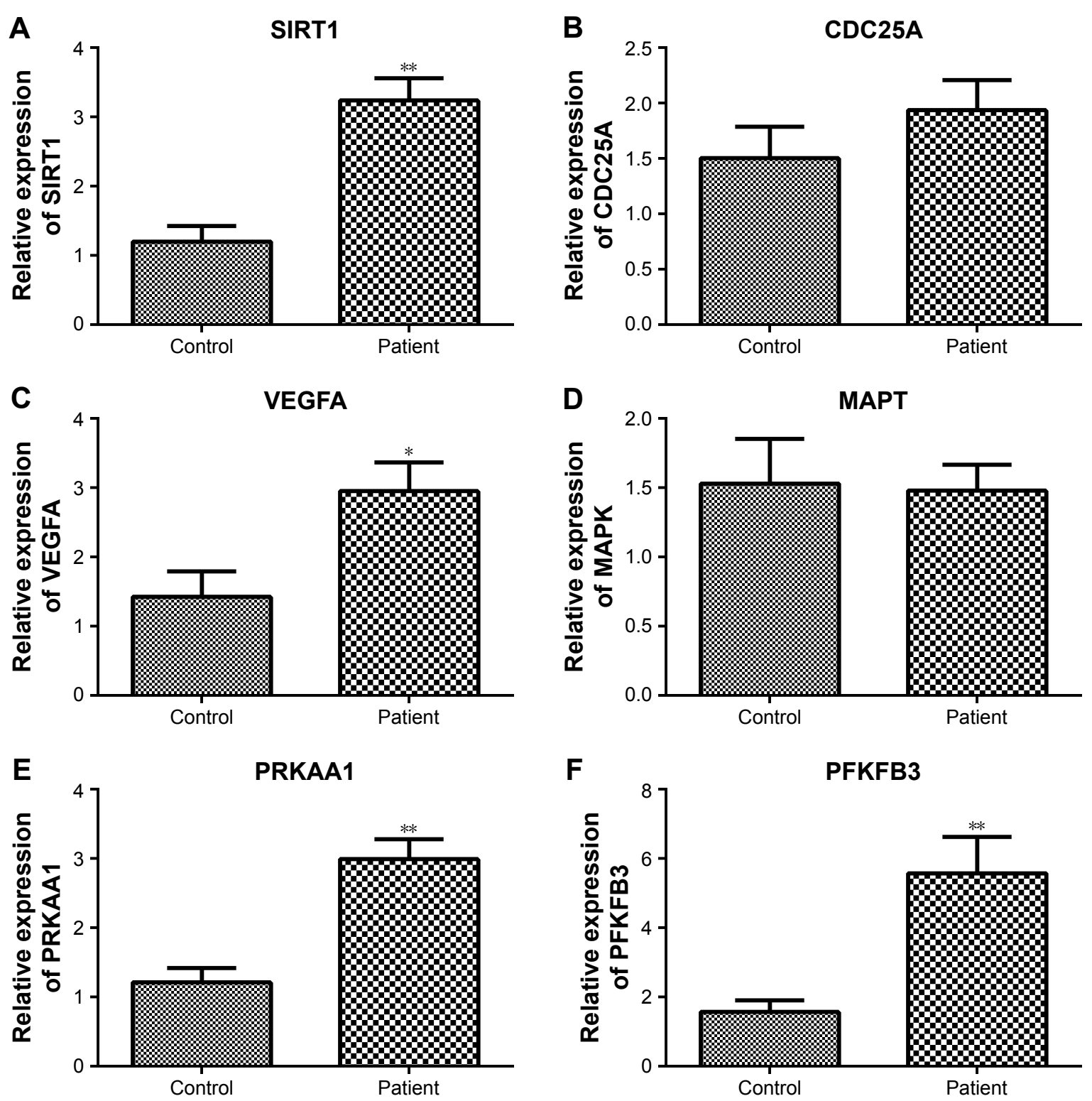

Figure 8 The expression of key target genes involved in cellular senescence (SIRTI and CDC25A), MAPK signaling pathway (VEGFA and MAPT), and AMPK signaling pathway (PRKAAI and PFKFB3).

Notes: (A) The relative expression of SITRI. (B) The relative expression of CDC25A. (C) The relative expression of VEGFA. (D) The relative expression of MAPT. (E) The relative expression of PRKAAI. (F) The relative expression of PFKFB3. $* P<0.05, * * P<0.01$.

the expression of VEGF, hinting the role of VEGF in agingrelated impairment of angiogenesis. ${ }^{33}$ Mohamed et $\mathrm{al}^{34}$ revealed that the activation of SIRT1 ameliorated skeletal muscle performance in pathophysiological conditions such as sarcopenia and disuse-induced atrophy in aging mice. Liao et $\mathrm{a}^{35}$ demonstrated that exercise, resveratrol, or their combination played a protective role in sarcopenia in aged rats through regulating AMPK/SIRT1 pathway. These observations suggest that our findings are significant.

Furthermore, in this study, hsa-miR-34a was also found to be closely related to endocrine resistance. It is found that the secondary hormones underlying insulin-like signaling may be involved in the regulation of aging in nematodes and flies. ${ }^{36}$ In all species, the endocrine system plays a key role in modulating the aging process. Although insulin resistance was found to accelerate muscle protein degradation, which causes muscle atrophy in old people, whether the endocrine system manipulated the aging process by insulin-like signaling remained to be ascertained. Thus, endocrine resistance may be an important event in the aging process regulated by hsa-miR-34a. Further studies are warranted in the future.

However, some limitations merit further consideration. First, whether the identified differentially expressed miRNAs can be used as muscle-specific miRNAs is still needed to be confirmed by more signature analysis. Second, our patient sample size was small, which might influence the stability 
of statistical power. More than five patient samples are required to be collected for validation of our results. Third, we detected the expression of four miRNAs in skeletal muscle from sarcopenic patients and control subjects. Nevertheless, the connection between aging and sarcopenia in this context was not investigated. In addition, the information on muscle mass or strength in relation to their original analysis was not provided, which hindering us for further patient analysis. Therefore, additional experiments and high throughput data are needed to confirm our findings.

\section{Conclusion}

miRNAs play regulatory roles in the aging process of skeletal muscles. AMPK signaling pathway, cellular senescence, and MAPK signaling pathway are significant pathways in the aging process. has-miR-19a and hsa-miR-34a, as significant nodes in miRNA regulatory network, may provide new perspectives to prevent muscle aging.

\section{Disclosure}

The authors report no conflicts of interest in this work.

\section{References}

1. Phillips SM. Nutritional supplements in support of resistance exercise to counter age-related sarcopenia. Adv Nutr. 2015;6(4):452-460.

2. Patel HP, Syddall HE, Jameson K, et al. Prevalence of sarcopenia in community-dwelling older people in the UK using the European Working Group on Sarcopenia in Older People (EWGSOP) definition: findings from the Hertfordshire Cohort Study (HCS). Age Ageing. 2013; 42(3):378-384.

3. Brown JC, Harhay MO, Harhay MN. Sarcopenia and mortality among a population-based sample of community-dwelling older adults. J Cachexia Sarcopenia Muscle. 2016;7(3):290-298.

4. Cannon JG. Cytokines in aging and muscle homeostasis. J Gerontol A Biol Sci Med Sci. 1995;50 Spec No:120-123.

5. Hagen JL, Krause DJ, Baker DJ, Fu MH, Tarnopolsky MA, Hepple RT. Skeletal muscle aging in F344BN F1-hybrid rats: I. Mitochondrial dysfunction contributes to the age-associated reduction in $\mathrm{VO}_{2}$ max. J Gerontol A Biol Sci Med Sci. 2004;59(11):1099-1110.

6. Jackson MJ. Skeletal muscle aging: role of reactive oxygen species. Crit Care Med. 2009;37(10 Suppl):S368-S371.

7. Weisleder N, Brotto M, Komazaki S, et al. Muscle aging is associated with compromised $\mathrm{Ca}^{2+}$ spark signaling and segregated intracellular $\mathrm{Ca}^{2+}$ release. J Cell Biol. 2006;174(5):639-645.

8. Hall JK, Banks GB, Chamberlain JS, Olwin BB. Prevention of muscle aging by myofiber-associated satellite cell transplantation. Sci Transl Med. 2010;2(57):57ra83.

9. Bhaumik D, Scott GK, Schokrpur S, et al. MicroRNAs miR-146a/b negatively modulate the senescence-associated inflammatory mediators IL-6 and IL-8. Aging. 2009;1(4):402-411.

10. Caygill EE, Johnston LA. Temporal regulation of metamorphic processes in Drosophila by the let-7 and miR-125 heterochronic microRNAs. Curr Biol. 2008;18(13):943-950.

11. Mcgregor RA, Poppitt SD, Cameron-Smith D. Role of microRNAs in the age-related changes in skeletal muscle and diet or exercise interventions to promote healthy aging in humans. Ageing Res Rev. 2014;17(9): $25-33$.
12. Murach KA, Mccarthy JJ. MicroRNAs, heart failure, and aging: potential interactions with skeletal muscle. Heart Fail Rev. 2016:1-10.

13. Mccarthy JJ. microRNA and skeletal muscle function: novel potential roles in exercise, diseases, and aging. Front Physiol. 2014;5(5): 290.

14. Zacharewicz E, della Gatta P, Reynolds J, et al. Identification of microRNAs linked to regulators of muscle protein synthesis and regeneration in young and old skeletal muscle. PLoS One. 2014;9(12):e114009.

15. Drummond MJ, Mccarthy JJ, Sinha M, et al. Aging and microRNA expression in human skeletal muscle: a microarray and bioinformatics analysis. Physiol Genomics. 2011;43(10):595-603.

16. Smyth GK. Limma: Linear models for microarray data. In: Gentleman R, Carey VJ, Huber W, Irizarry RA, Dudoit S, editors. Bioinformatics and Computational Biology Solutions Using R and Bioconductor. New York: Springer; 2005:397-420.

17. Xiao F, Zuo Z, Cai G, Kang S, Gao X, Li T. miRecords: an integrated resource for microRNA-target interactions. Nucleic Acids Res. 2009;37(Database issue):D105-D110.

18. Shannon P, Markiel A, Ozier O, et al. Cytoscape: a software environment for integrated models of biomolecular interaction networks. Genome Res. 2003;13(11):2498-2504.

19. Ashburner M, Ball CA, Blake JA, et al. Gene Ontology: tool for the unification of biology. Nat Genet. 2000;25(1):25-29.

20. Kanehisa M, Goto S. KEGG: Kyoto Encyclopedia of Genes and Genomes. Nucleic Acids Res. 2000;28(1):27-30.

21. Huang Daw, Sherman BT, Lempicki RA. Systematic and integrative analysis of large gene lists using DAVID bioinformatics resources. Nat Protoc. 2009;4(1):44-57.

22. Huang Daw, Sherman BT, Lempicki RA. Bioinformatics enrichment tools: paths toward the comprehensive functional analysis of large gene lists. Nucleic Acids Res. 2009;37(1):1-13.

23. Yu G, Wang LG, Han Y, He QY. clusterProfiler: an R package for comparing biological themes among gene clusters. OMICS. 2012; 16(5):284-287.

24. Szklarczyk D, Franceschini A, Wyder S, et al. STRING v10: proteinprotein interaction networks, integrated over the tree of life. Nucleic Acids Res. 2015;43(Database issue):D447-52-D452-52.

25. Bandettini WP, Kellman P, Mancini C, et al. MultiContrast Delayed Enhancement (MCODE) improves detection of subendocardial myocardial infarction by late gadolinium enhancement cardiovascular magnetic resonance: a clinical validation study. J Cardiovasc Magn Reson. 2012;14:83.

26. Janky R, Verfaillie A, Imrichová H, et al. iRegulon: from a gene list to a gene regulatory network using large motif and track collections. PLoS Comput Biol. 2014;10(7):e1003731.

27. Salminen A, Kaarniranta K, Kai K. AMP-activated protein kinase (AMPK) controls the aging process via an integrated signaling network. Ageing Res Rev. 2012;11(2):230-241.

28. Reznick RM, Zong H, Li J, et al. Aging-associated reductions in AMP-activated protein kinase activity and mitochondrial biogenesis. Cell Metab. 2007;5(2):151-156.

29. $\mathrm{Wu} \mathrm{W}, \mathrm{Xu} \mathrm{Z}$, Zhang L, et al. Muscle-specific deletion of Prkaa1 enhances skeletal muscle lipid accumulation in mice fed a high-fat diet. J Physiol Biochem. 2018;74(2):195-205.

30. Collado M, Blasco MA, Serrano M, Chavent M. Cellular senescence in cancer and aging. Cell. 2007;130(2):223-233.

31. Kramer HF, Goodyear LJ. Exercise, MAPK, and NF-kappaB signaling in skeletal muscle. J Appl Physiol. 2007;103(1):388-395.

32. Bernet JD, Doles JD, Hall JK, Kelly Tanaka K, Carter TA, Olwin BB. p38 MAPK signaling underlies a cell-autonomous loss of stem cell self-renewal in skeletal muscle of aged mice. Nat Med. 2014;20(3): 265-271.

33. Deng X, Ahluwalia A, Xiong X, Szabo S, Sandor Z, Tarnawski A. S1619 MMP9-mediated upregulation of endostatin and downregulation of VEGF in aging gastric mucosa: novel mechanism for impaired angiogenesis. Gastroenterology. 2008;134(4):A-236. 
34. Mohamed JS, Wilson JC, Myers MJ, Sisson KJ, Alway SE. Dysregulation of SIRT-1 in aging mice increases skeletal muscle fatigue by a PARP-1-dependent mechanism. Aging. 2014;6(10):820-834.

35. Liao ZY, Chen JL, Xiao MH, et al. The effect of exercise, resveratrol or their combination on Sarcopenia in aged rats via regulation of AMPK Sirt1 pathway. Exp Gerontol. 2017;98:177-183.
36. Tatar M, Bartke A, Antebi A. The endocrine regulation of aging by insulin-like signals. Science. 2003;299(5611):1346-1351.

\section{Publish your work in this journal}

Clinical Interventions in Aging is an international, peer-reviewed journal focusing on evidence-based reports on the value or lack thereof of treatments intended to prevent or delay the onset of maladaptive correlates of aging in human beings. This journal is indexed on PubMed Central, MedLine,

\section{Dovepress}

CAS, Scopus and the Elsevier Bibliographic databases. The manuscript management system is completely online and includes a very quick and fair peer-review system, which is all easy to use. Visit http://www.dovepress. $\mathrm{com} /$ testimonials.php to read real quotes from published authors. 